\title{
The use of generation stochastic models to study an epidemic disease
}

\author{
S Seddighi Chaharborj ${ }^{1,2,3^{*}}$, I Fudziah', MR Abu Bakar ${ }^{1}$, R Seddighi Chaharborj ${ }^{4}$, ZA Majid ${ }^{1,5}$ and \\ AGB Ahmad ${ }^{6}$
}

*Correspondence:

sseddighi2007@yahoo.com

'Department of Mathematics,

Faculty of Science, Universiti Putra

Malaysia, 43400 UPM, Selangor,

Malaysia

2 Plasma Physics and Nuclear Fusion

Research School, Nuclear Science

and Technology Research Institute (NSTRI), P.O. Box 14395-836, Tehran,

Iran

Full list of author information is

available at the end of the article

\begin{abstract}
Stochastic models have an important role in modeling and analyzing epidemic diseases for small size population. In this article, we study the generation of stochastic models for epidemic disease susceptible-infective-susceptible model. Here, we use the separation variable method to solve partial differential equation and the new developed modified probability generating function (PGF) of a random process to include a random catastrophe to solve the ordinary differential equations generated from partial differential equation. The results show that the probability function is too sensitive to $\mu, \beta$ and $\gamma$ parameters.
\end{abstract}

Keywords: epidemic diseases; susceptible-infective-susceptible; deterministic model; stochastic model; probability function

\section{Deterministic susceptible-infective-susceptible model}

Figure 1 shows a deterministic susceptible-infective-susceptible model for an epidemic disease. In this figure, $S$ is the susceptible population, $I$ is the infective population, $\mu>$ 0 is the natural death rate, $\gamma>0$ is the removal rate which is a constant. Note that $S, I \geq 0$ because they represent the number of people. The infection rate, $\lambda$, depends on the number of partners per individual per unit time $(r>0)$ and the transmission probability per partner $(\beta>0)$. In this system, the first susceptible population in class $S$ is going to be infected, then infected population in class $I$ is going to be susceptible again. The following system of ODE's describes this susceptible-infective-susceptible model [1]

$$
\left\{\begin{array}{l}
\frac{d S(t)}{d t}=\gamma I(t)-\mu-\lambda S(t), \\
\frac{d I(t)}{d t}=\lambda S(t)-\mu-\gamma .
\end{array}\right.
$$

Figure 1 illustrates the system (1). This system is nonlinear due to the form of $\lambda=\beta I$.

\section{Generation stochastic susceptible-infective-susceptible model}

In this section, we present the state of the generation stochastic [2-5] susceptibleinfective-susceptible model. The stochastic susceptible-infective-susceptible model is similar to the deterministic susceptible-infective-susceptible model, for the deterministic model we can find an exact function but for the stochastic model, we cannot obtain

(c) 2013 Seddighi Chaharborj et al: licensee Springer. This is an Open Access article distributed under the terms of the Creative Commons Attribution License (http://creativecommons.org/licenses/by/2.0), which permits unrestricted use, distribution, and reproduction in any medium, provided the original work is properly cited. 


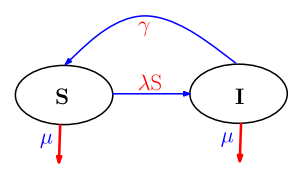

Figure 1 A schematic of system (1).

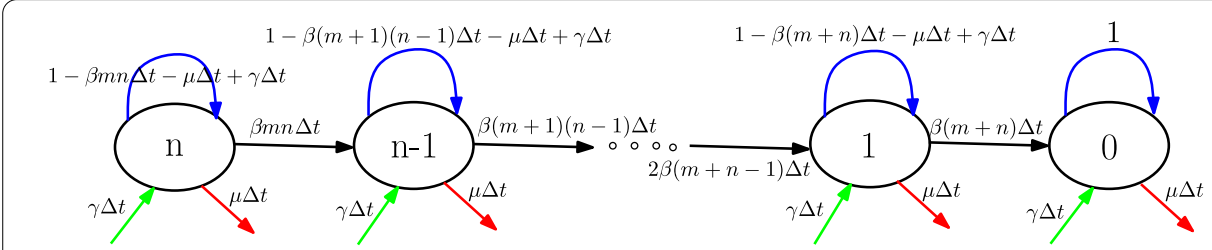

Figure 2 Stochastic susceptible-infective-susceptible model state diagram.

Table 1 Transition diagram for the stochastic susceptible-infective-susceptible model

\begin{tabular}{ll}
\hline Transitions & Rates \\
\hline$i \rightarrow i-1$ & $\beta i(m+(n-i))+\mu-\gamma$ \\
$i \rightarrow 0$ & $\mu-\gamma$ \\
& for $i=n, n-1, n-2, \ldots, 2,1$ \\
\hline
\end{tabular}

In this figure, $\mu>0$ is the natural death rate, $\gamma>0$ is the removal rate which is a constant and $\beta>0$ is the transmission probability per partner.

an exact function. Figure 2 shows the state diagram for the stochastic [2-5] susceptibleinfective-susceptible model [1, 6-10].

At $t_{0}$, if $m$ is infective and $n$ is susceptible, namely $S\left(t_{0}\right)+I\left(t_{0}\right)=n+m$, then $P_{i}(t)=$ $P[S(t)=i \mid S(0)=n]$ is the probability function in the time $t$ and stage $i$. Here, our goal is to determine $P_{i}(t)$. Table 1 shows the transition diagram for this model. To determine $P_{i}(t)$ 's, we should create the Kolmogorov equations. From Figure 2, we have

$$
P[\text { staying at state } i]=1-(\beta i(m+(n-i)) \Delta t+\mu \Delta t-\gamma \Delta t)
$$

and

$$
P[\text { moving from state } i \text { to } i-1]=\beta i(m+(n-i)) \Delta t+\mu \Delta t-\gamma \Delta t \text {. }
$$

Now, to produce the forward Kolmogorov equations, we have

$$
\begin{aligned}
P_{i}(t+\Delta t)= & P(0 \text { contact during } \Delta t \mid S(t)=i) P_{i}(t) \\
& +P(1 \text { contact during } \Delta t \mid S(t)=i+1) P_{i+1}(t) \\
= & P(\text { staying at state } i) P_{i}(t)+P(\text { moving from state } i+1 \text { to } i) P_{i+1}(t) \\
= & (1-(\beta i(m+(n-i)) \Delta t+\mu \Delta t-\gamma \Delta t)) P_{i}(t) \\
& +(\beta(i+1)(m+(n-i-1)) \Delta t+\mu \Delta t-\gamma \Delta t) P_{i+1}(t) .
\end{aligned}
$$


So,

$$
\begin{aligned}
P_{i}(t+\Delta t)-P_{i}(t)= & -(\beta i(m+n-i) \Delta t+\mu \Delta t-\gamma \Delta t) P_{i}(t) \\
& +(\beta(i+1)(m+n-i-1) \Delta t+\mu \Delta t \\
& -\gamma \Delta t) P_{i+1}(t),
\end{aligned}
$$

then

$$
\begin{aligned}
\frac{P_{i}(t+\Delta t)-P_{i}(t)}{\Delta t}= & -(\beta i(m+n-i)+\mu-\gamma) P_{i}(t) \\
& +(\beta(i+1)(m+n-i-1)+\mu-\gamma) P_{i+1}(t) .
\end{aligned}
$$

Having limited from both sides of Eq. (5), when $\Delta t \rightarrow 0$, we have

$$
\begin{aligned}
P_{i}^{\prime}(t)= & \lim _{\Delta t \rightarrow 0} \frac{P_{i}(t+\Delta t)-P_{i}(t)}{\Delta t}=-(\beta i(m+n-i)+\mu-\gamma) P_{i}(t) \\
& +(\beta(i+1)(m+n-i-1)+\mu-\gamma) P_{i+1}(t) .
\end{aligned}
$$

Therefore, the forward Kolmogorov equations for this model will be as follows:

$$
\begin{aligned}
P_{i}^{\prime}(t)= & -(\beta i(m+n-i)+\mu-\gamma) P_{i}(t) \\
& +(\beta(i+1)(m+n-i-1)+\mu-\gamma) P_{i+1}(t) .
\end{aligned}
$$

The probabilities function $P_{i}(t)$ is found from Eq. (7). Also, from probability generating functions (PGFs) and partial differential functions equations (PDEs), the probabilities function $P_{i}(t)$ can be obtained. Probability generating functions can be written as

$$
y(x, t)=\sum_{i=0}^{n} P_{i}(t) x^{i}=P_{0}(t)+P_{1}(t) x+P_{2}(t) x^{2}+\cdots+P_{n}(t) x^{n} .
$$

Now, the partial derivative of $y(x, t)$ with respect to $t$ will be $\frac{\partial y}{\partial t}=\sum_{i=0}^{n} P_{i}^{\prime}(t) x^{i}$, so one can write the partial derivative as follows:

$$
\begin{aligned}
\frac{\partial y}{\partial t}= & \sum_{i=0}^{n}\left[-(\beta i(m+n-i)+\mu-\gamma) P_{i}(t)\right. \\
& \left.+(\beta(i+1)(m+n-i-1)+\mu-\gamma) P_{i+1}(t)\right] x^{i} .
\end{aligned}
$$

Having simplified Eq. (9), we can write

$$
\begin{aligned}
\frac{\partial y(x, t)}{\partial t}= & (\mu-\gamma) \cdot \frac{1}{x}+(\gamma-\mu) y(x, t)+\beta(m+n-1)(1-x) \frac{\partial y(x, t)}{\partial x} \\
& +\beta x(x-1) \frac{\partial^{2} y(x, t)}{\partial x^{2}} .
\end{aligned}
$$


The separation variable method is employed to solve Eq. (10). If $y(x, t)=X(x) T(t)$, then we have

$$
\begin{aligned}
\frac{T^{\prime}(t)}{T(t)}= & (\gamma-\mu)+(\mu-\gamma) \cdot \frac{1}{x} \cdot \frac{1}{X(x)}+\beta(m+n-1)(1-x) \frac{X^{\prime}(x)}{X(x)} \\
& +\beta x(x-1) \frac{X^{\prime \prime}(x)}{X(x)} .
\end{aligned}
$$

Two sides of Eq. (11) are equal, so

$$
\begin{aligned}
\frac{T^{\prime}(t)}{T(t)}= & -c \text { and }(\gamma-\mu)+(\mu-\gamma) \cdot \frac{1}{x} \cdot \frac{1}{X(x)}+\beta(m+n-1)(1-x) \frac{X^{\prime}(x)}{X(x)} \\
& +\beta x(x-1) \frac{X^{\prime \prime}(x)}{X(x)}=-c .
\end{aligned}
$$

To include a random catastrophe presented by Gani and Swift in 2006 [11], we develop the modified probability generating function (PGF) of a random process to solve Eq. (10) as follows:

$$
G(x, t)=\sum_{j=0}^{n} G_{j}(x, t)=\sum_{j=0}^{n}\left[e^{-(\mu-\gamma) t} y(x, t)+\int_{0}^{t}(\mu-\gamma) e^{-(\mu-\gamma) v} y(x, v) d v\right],
$$

with $\mu-\gamma \geq 0$, here $y(x, t)$ is the answer of Eq. (10) when $\mu=0, \gamma=0$. So, we can put $\mu=0, \gamma=0$ with $m=1$ in Eq. (12)

$$
x(x-1) X^{\prime \prime}(x)-n(x-1) X^{\prime}(x)+(c / \beta) X(x)=0 .
$$

This equation was solved by Bailey in 1963 [12]. After having solved Eq. (14) by Maple, we have

$$
\begin{aligned}
X(x)= & -C_{1} \times{ }_{2} F_{1}\left[-1 / 2 \frac{\sqrt{\beta+2 \beta n+n^{2} \beta-4 c}+\sqrt{\beta}(-1+n)}{\sqrt{\beta}},\right. \\
& \left.-1 / 2 \frac{-\sqrt{\beta+2 \beta n+n^{2} \beta-4 c}+\sqrt{\beta}(-1+n)}{\sqrt{\beta}} ;-n ; x\right],
\end{aligned}
$$

where ${ }_{2} F_{1}[\cdot, \cdot ; ; \cdot \cdot]$ is a hypergeometric function.

Notation 1 The standard hypergeometric function ${ }_{2} F_{1}[a, b ; c ; x]$ is as follows:

$$
{ }_{2} F_{1}[a, b ; c ; x]=\sum_{i=0}^{\infty} \frac{(a)_{i}(b)_{i}}{(c)_{i}} \times \frac{x^{i}}{i !}
$$

where $(a)_{i}=a(a+1)(a+2)(a+3) \cdots(a+i-1)$ with $(a)_{0}=1$ is the Pochhammer symbol. The derivatives of ${ }_{2} F_{1}[a, b ; c ; x]$ are given by

$$
\begin{aligned}
& \frac{d_{2} F_{1}[a, b, c, x]}{d x}=\frac{a b}{c}{ }_{2} F_{1}[a+1, b+1, c+1, x], \\
& \frac{d^{2}{ }_{2} F_{1}[a, b, c, x]}{d x^{2}}=\frac{a(a+1) b(b+1)}{c(c+1)}{ }_{2} F_{1}[a+2, b+2, c+2, x],
\end{aligned}
$$




$$
\frac{d^{3}{ }_{2} F_{1}[a, b, c, x]}{d x^{3}}=\frac{a(a+1)(a+2) b(b+1)(b+2)}{c(c+1)(c+2)}{ }_{2} F_{1}[a+3, b+3, c+3, x]
$$

Also, from equation $\frac{T^{\prime}(t)}{T(t)}=-c$, we have $T(t)=k_{1} e^{-c t}$. So,

$$
\begin{aligned}
y(x, t)= & e^{-c t} \frac{1}{{ }_{2} F_{1}\left[-1 / 2 \frac{\sqrt{\beta+2 \beta n+n^{2} \beta-4 c}+\sqrt{\beta}(-1+n)}{\sqrt{\beta}},-1 / 2 \frac{-\sqrt{\beta+2 \beta n+n^{2} \beta-4 c}+\sqrt{\beta}(-1+n)}{\sqrt{\beta}} ;-n ; 1\right]} \\
& \times{ }_{2} F_{1}\left[-1 / 2 \frac{\sqrt{\beta+2 \beta n+n^{2} \beta-4 c}+\sqrt{\beta}(-1+n)}{\sqrt{\beta}},\right. \\
& \left.-1 / 2 \frac{-\sqrt{\beta+2 \beta n+n^{2} \beta-4 c}+\sqrt{\beta}(-1+n)}{\sqrt{\beta}} ;-n ; x\right] .
\end{aligned}
$$

In Eq. (17) we can take $c=j(N+m-j) \beta ; N=n+\epsilon$, here $\epsilon$ is so small parameter. Then from Eq. (17) and Eq. (13), one can write

$$
\begin{aligned}
G(x, t)= & \sum_{j=0}^{n}\left[e^{-(\mu-\gamma) t} \times e^{-j(N+m-j) \beta t} \frac{1}{{ }_{2} F_{1}[-j, j-N-1,-N, 1]}\right. \\
& \times{ }_{2} F_{1}[-j, j-N-1,-N, x]+\int_{0}^{t}(\mu-\gamma) e^{-(\mu-\gamma) v} \times e^{-j(m+N-j) \beta v} \\
& \left.\times \frac{1}{{ }_{2} F_{1}[-j, j-N-1,-N, 1]} \times{ }_{2} F_{1}[-j, j-N-1,-N, \nu] d \nu\right]
\end{aligned}
$$

Thus,

$$
\begin{aligned}
G(x, t)= & \sum_{j=0}^{n} \lambda_{j} \times{ }_{2} F_{1}[-j, j-N-1,-N, x] \times\left[\left(e^{-[(\mu-\gamma)+j(N+m-j) \beta] t}\right.\right. \\
& \left.+\int_{0}^{t}(\mu-\gamma) e^{-[(\mu-\gamma)+j(N+m-j) \beta)] v} d v\right] \\
= & \sum_{j=0}^{n} \lambda_{j} \times{ }_{2} F_{1}[-j, j-N-1,-N, x] \times\left[e^{-[(\mu-\gamma)+j(m+N-j) \beta] t}\right. \\
& \left.-\frac{1-\mathrm{e}^{\left(-\mu+\gamma+j \beta N-j^{2} \beta+j \beta\right) t}}{-\mu+\gamma+j \beta N-j^{2} \beta+j \beta}\right]
\end{aligned}
$$

where

$$
\lambda_{j}=\frac{(-1)^{j} n !(N-2 j+1) N ! \Gamma(-N+j-1)}{j !(n-j) !(N-n) !(-1)^{n+1} \Gamma(n-N+j)} .
$$

Now, to find $P_{0}(t), P_{1}(t), P_{2}(t), \ldots, P_{k}(t)$, one can calculate $G(x, t)$ as follows:

$$
G(x, t)=\sum_{k=0}^{\infty} P_{k}(t) x^{k}=P_{0}(t)+P_{1}(t) x+P_{2}(t) x^{2}+\cdots
$$


So,

$$
\begin{aligned}
P_{0}(t)= & G(0, t) \\
= & \sum_{j=0}^{n} \lambda_{j} \times\left[e^{-[(\mu-\gamma)+j(m+N-j) \beta)] t}\right. \\
& \left.-\frac{1-e^{\left(-\mu+\gamma+j \beta N-j^{2} \beta+j \beta\right) t}}{-\mu+\gamma+j \beta N-j^{2} \beta+j \beta}\right] .
\end{aligned}
$$

Hence, $P_{1}(t)$ and $P_{2}(t)$ are obtained as follows:

$$
\begin{aligned}
P_{1}(t)= & \left.\frac{d G(x, t)}{d x}\right|_{x=0}=\sum_{j=0}^{n} \frac{1}{2 !} \frac{(-j)(j-N-1)}{(-N)} \times \lambda_{j} \times \Psi_{j}(t), \\
P_{2}(t)= & \left.\frac{d^{2} G(x, t)}{d x^{2}}\right|_{x=0}=\sum_{j=0}^{n} \frac{1}{3 !} \frac{(-j)(-j+1)(j-N-1)(j-N-1+1)}{(-N)(-N+1)} \\
& \times \lambda_{j} \times \Psi_{j}(t),
\end{aligned}
$$

Therefore,

$$
P_{k}(t)=\left.\frac{d^{k} G(x, t)}{d x^{k}}\right|_{x=0}=\sum_{j=0}^{n} \frac{1}{k !} \frac{(-j)_{k}(j-N-1)_{k}}{(-N)_{k}} \times \lambda_{j} \times \Psi_{j}(t),
$$

with

$$
\Psi_{j}(t)=\left[e^{-j \mu t-(n-j) \gamma t-\left(m+N-j^{2}\right) \beta t}-\frac{1-e^{\left(-\mu+\gamma+j \beta N-j^{2} \beta+j \beta\right) t}}{-\mu+\gamma+j \beta N-j^{2} \beta+j \beta}\right] .
$$

\section{Numerical results}

Some numerical examples illustrate the behavior of the probability function $P_{K}(t, \beta, \mu, \gamma)$ with the following parameters: $t=1 ; \beta=0.3 ; \mu=0.3 ; \gamma=0.1$.

Figure 3(a) and (b) show the behavior of the probability functions, $P_{0}(t, \beta)$ and $P_{8}(t, \beta)$ with $\mu=0.3$ and $\gamma=0.1$ when $0<t<5$ and $0<\beta<1$.

Figure 3(a) shows that with an increase in $t$ and $\beta$, the probability function $P_{0}(t, \beta)$ increases fast, but in Figure 3(b) $P_{8}(t, \beta)$ decreases fast.

Figure 4(a) and (b) show the behavior of the probability functions, $P_{0}(t, \mu)$ and $P_{8}(t, \mu)$ with $\beta=0.3$ and $\gamma=0.1$ when $0<t<5$ and $0<\mu<1$. Figure 4(a) shows that as $t$ increases, the probability function $P_{0}(t, \mu)$ increases, but with an increase in $\mu$, the probability function $P_{0}(t, \mu)$ decreases. Figure 4 (b) shows that as $t$ increases, the probability function $P_{8}(t, \mu)$ decreases, but with an increase in $\mu$, the probability function $P_{0}(t, \mu)$ increases.

Figure 5(a) and (b) display the probability functions, $P_{0}(t, \gamma)$ and $P_{8}(t, \gamma)$ with $\beta=0.3$ and $\mu=0.3$. From Figure 5(a) when $0<t<1$ and $0<\gamma<1$, the probability function $P_{0}(t, \gamma)$ 


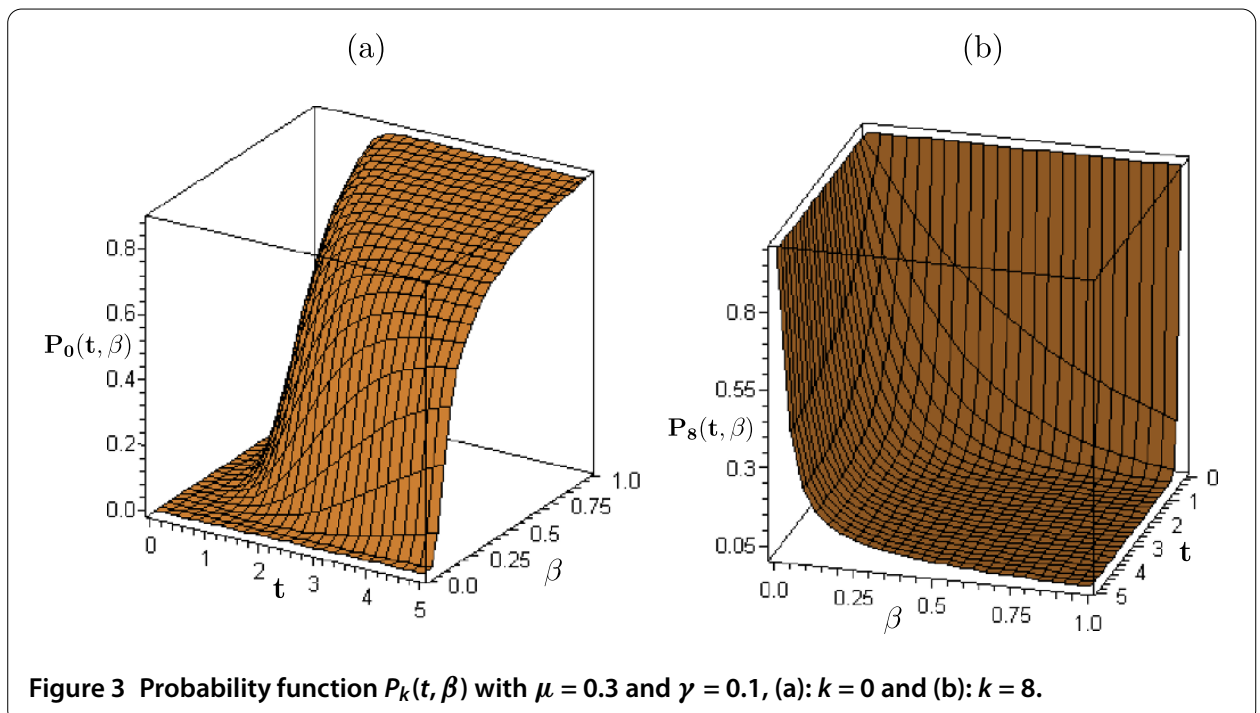

(a)

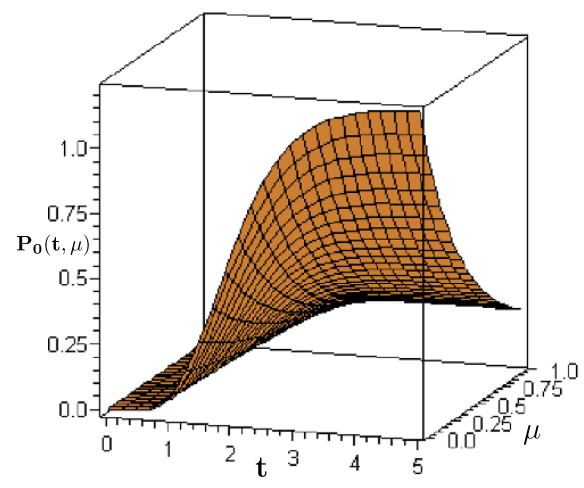

(b)

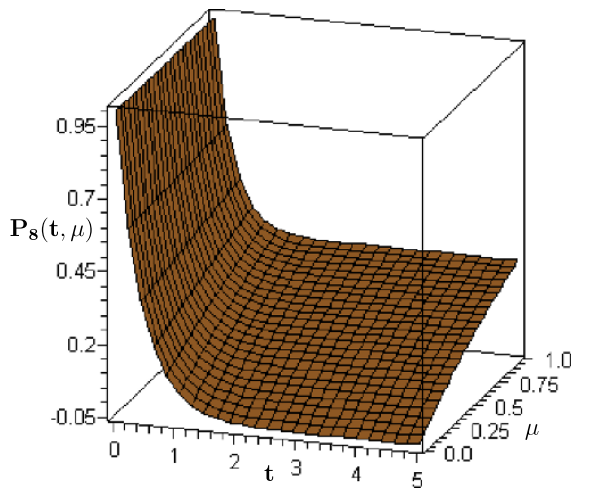

Figure 4 Probability function $P_{k}(t, \mu)$ with $\beta=0.3$ and $\gamma=0.1$, (a): $k=0$ and $(b): k=8$.

(a)

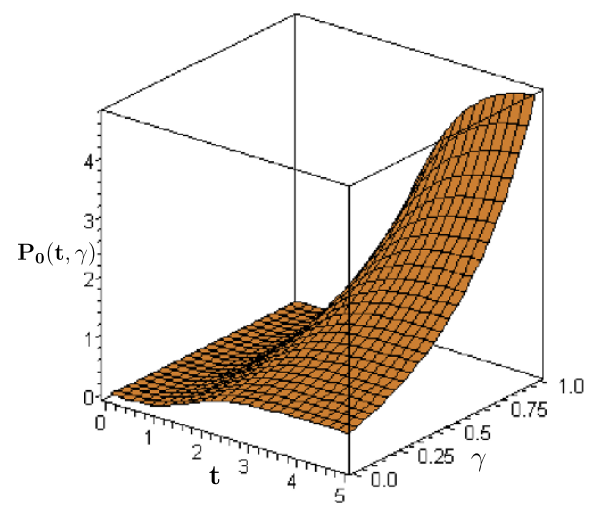

(b)

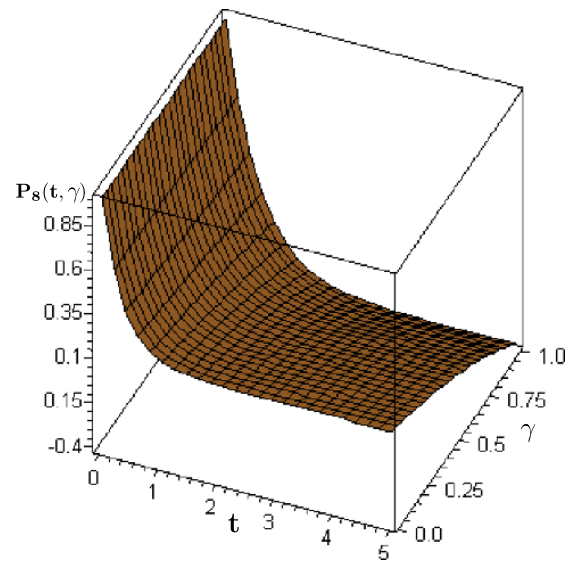

Figure 5 Probability function $P_{k}(t, \gamma)$ with $\beta=0.3$ and $\mu=0.3$, (a): $k=0$ and (b): $k=8$. 

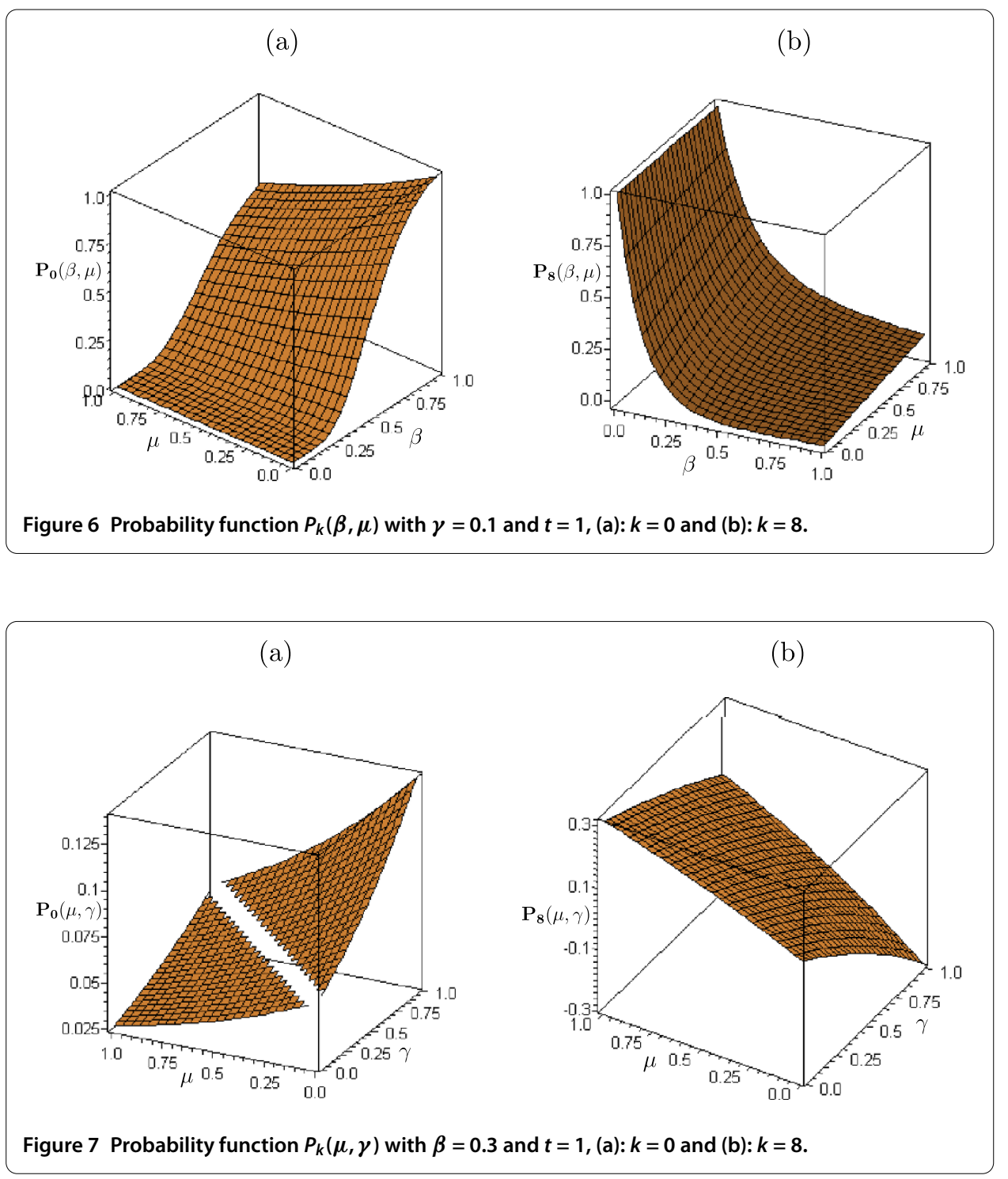

is nearly zero, but for $1<t<5$ as $t$ increases, $P_{0}(t, \gamma)$ slowly increases, and as $\gamma$ increases, $P_{0}(t, \gamma)$ sharply increases. In Figure 5(b), $P_{8}(t, \gamma)$ is almost constant for $1<t<5$, but with a decrease in $\gamma$ from 1 to $0, P_{8}(t, \gamma)$ increases; also, Figure 5(b) shows the highest value of $P_{8}(t, \gamma)$ when $0<t<1$ and $0<\gamma<1$.

Figure 6(a) and (b) show the probability functions, $P_{0}(\beta, \mu)$ and $P_{8}(\beta, \mu)$ with $\gamma=0.1$ and $t=1$. Figure 6 (a) depicts that for $0<\beta<1$, as $\mu$ increases from 0 to 1 , the probability function $P_{0}(\beta, \mu)$ increases. Figure 6(b) shows that for $0<\mu<1$, with an increase in $\beta$ and $\mu$, the probability function $P_{8}(\beta, \mu)$ increases.

Figure 7(a) and (b) illustrate the probability functions, $P_{0}(\mu, \gamma)$ and $P_{8}(\mu, \gamma)$ with $\beta=0.3$ and $t=1$. Figure 7(a) shows that the probability function $P_{0}(\mu, \gamma)$ decreases with an increase in $\mu$, but inversely it increases with an increase in $\gamma$. In Figure 7(a) we observe a separation which means that in the probability function $P_{K}(\mu, \gamma)$ we have $\mu \neq \gamma$. Figure 7(b) depicts that the probability function $P_{8}(\mu, \gamma)$ increases when $\mu$ increases, although it decreases with an increase in $\gamma$. 


\section{Conclusions}

We have presented the generation of a stochastic model for the susceptible-infectivesusceptible model. The separation variable method has been applied to solve a partial differential equation of this generation. So, two ordinary differential equations have been achieved which relate to the parameter $x$. To solve this equation, we used the developed modified probability generating function (PGF) of a random process to consider a random catastrophe. Numerical results showed the behavior of the probability function $P_{k}(t, \beta, \mu, \gamma)$ when $0<t<5$ and $0<\beta, \mu, \gamma<1$.

\section{Competing interests}

The authors declare that they have no competing interests.

\section{Authors' contributions}

All authors carried out the proof and conceived of the study. All authors read and approved the final manuscript.

\section{Author details}

${ }^{1}$ Department of Mathematics, Faculty of Science, Universiti Putra Malaysia, 43400 UPM, Selangor, Malaysia. ${ }^{2}$ Plasma Physics and Nuclear Fusion Research School, Nuclear Science and Technology Research Institute (NSTRI), P.O. Box 14395-836, Tehran, Iran. ${ }^{3}$ Department of Mathematics, Science and Research Branch, Islamic, Azad University, Bushehr Branch, Bushehr, Iran. ${ }^{4}$ Department of Applied Mathematics and Computer Science, Eastern Mediterranean University, Famagusta, Northern Cyprus. ${ }^{5}$ Institute of Mathematical Research, Universiti Putra Malaysia, 43400 UPM Serdang Selangor Darul Ehsan, Selangor, Malaysia. ${ }^{6}$ School of Mathematical, Faculty of Science and Technology, Universiti Kebangsaan Malaysia, 43600 UKM Bangi, Selangor, Malaysia.

\section{Acknowledgements}

The authors thank the referees for valuable comments and suggestions which improved the presentation of this manuscript.

\section{Received: 28 October 2012 Accepted: 18 December 2012 Published: 9 January 2013}

\section{References}

1. Hethcote, HW: The mathematics of infectious diseases. SIAM Rev. 42, 599 (2000)

2. Tomasz, RB, Jacek, J, Mariusz, N: Study of dependence for some stochastic processes: symbolic Markov copulae. Stoch. Process. Appl. 122, 930-951 (2012)

3. Nathalie, E: Stochastic order for alpha-permanental point processes. Stoch. Process. Appl. 122, 952 (2012)

4. Said, $H$, Jianfeng, Z: Switching problem and related system of reflected backward SDEs. Stoch. Process. Appl. 120, 403 (2010)

5. Richard, AD, Li, S: Functional convergence of stochastic integrals with application to statistical inference. Stoch. Process. Appl. 122, 725 (2012)

6. Seddighi Chaharborj, S, Abu Bakar, MR, Fudziah, I: Study of stochastic systems for epidemic disease models. Int J. Mod. Phys.: Conf. Ser. 9, 373-379 (2012)

7. Seddighi Chaharborj, S, Gheisari, Y: Study of reproductive number in epidemic disease modeling. Adv. Stud. Biol. 3, 267-271 (2011)

8. Seddighi Chaharbor, S, Gheisari, Y: Study of reproductive number in SIR-SI model. Adv. Stud. Biol. 3, 309-317 (2011)

9. Seddighi Chaharborj, S, Abu Bakar, MR, Fudziah, I, Noor Akma, I, Malik, AH, Alli, V: Behavior stability in two SIR-style models for HIV. Int. J. Math. Anal. 4, 427-434 (2010)

10. Seddighi Chaharborj, S, Abu Bakar, MR, Malik, AH, Mehrkanoon, S: Solving the SI model for HIV with the homotopy perturbation method. Int. J. Math. Anal. 22, 211-218 (2009)

11. Swift, RJ, Gani, J: A simple approach to birth processes with random catastrophes. J. Comb. Inf. Syst. Sci. 31, 325-331 (2006)

12. Norman, TJB: The simple stochastic epidemic: a complete solution in terms of known functions. Biometrika 50, 235 (1963)

doi:10.1186/1687-1847-2013-7

Cite this article as: Seddighi Chaharborj et al.: The use of generation stochastic models to study an epidemic disease. Advances in Difference Equations 2013 2013:7. 